\title{
The solar carbon, nitrogen, and oxygen abundances from a 3D LTE analysis of molecular lines ${ }^{\star}$
}

\author{
A. M. Amarsi ${ }^{1}$, N. Grevesse ${ }^{2,3}$, M. Asplund, and R. Collet ${ }^{4}$ \\ 1 Theoretical Astrophysics, Department of Physics and Astronomy, Uppsala University, Box 516, SE-751 20 Uppsala, Sweden \\ e-mail: anish.amarsi@physics.uu.se \\ 2 Centre Spatial de Liège, Université de Liége, avenue Pré Aily, B-4031 Angleur-Liège, Belgium \\ 3 Space sciences, Technologies and Astrophysics Research (STAR) Institute, Université de Liège, Allée du 6 août, 17, B5C, B-4000 \\ Liège, Belgium \\ ${ }^{4}$ Stellar Astrophysics Centre, Department of Physics and Astronomy, Aarhus University, Ny Munkegade 120, DK-8000 Aarhus C, \\ Denmark
}

Received / Accepted

\begin{abstract}
Carbon, nitrogen, and oxygen are the fourth, sixth, and third most abundant elements in the Sun. Their abundances remain hotly debated due to the so-called solar modelling problem that has persisted for almost 20 years. We revisit this issue by presenting a homogeneous analysis of 408 molecular lines across 12 diagnostic groups, observed in the solar intensity spectrum. Using a realistic $3 \mathrm{D}$ radiative-hydrodynamic model solar photosphere and LTE (local thermodynamic equilibrium) line formation, we find log $\epsilon_{\mathrm{C}}=$ $8.47 \pm 0.02, \log \epsilon_{\mathrm{N}}=7.89 \pm 0.04$, and $\log \epsilon_{\mathrm{O}}=8.70 \pm 0.04$. The stipulated uncertainties mainly reflect the sensitivity of the results to the model atmosphere; this sensitivity is correlated between the different diagnostic groups, which all agree with the mean result to within 0.03 dex. For carbon and oxygen, the molecular results are in excellent agreement with our 3D non-LTE analyses of atomic lines. For nitrogen, however, the molecular indicators give a 0.12 dex larger abundance than the atomic indicators, and our best estimate of the solar nitrogen abundance is given by the mean: 7.83 dex. The solar oxygen abundance advocated here is close to our earlier determination of $8.69 \mathrm{dex}$, and so the present results do not significantly alleviate the solar modelling problem.
\end{abstract}

Key words. radiative transfer — line: formation — Sun: abundances — Sun: photosphere — Sun: atmosphere

\section{Introduction}

Carbon, nitrogen, and oxygen are among the most important elements in astrophysics today. This is especially true in the context of our solar system, where they are the fourth, sixth, and third most abundant elements respectively (Asplund et al. 2021). Combined, they dominate the metal content of the solar interior; as such their abundances are central to the debate on the long-standing so-called solar modelling problem (see e.g. Bahcall et al. 2005; Basu \& Antia 2008; Buldgen et al. 2019; Christensen-Dalsgaard 2021). This describes an obstinate discrepancy between helioseismic inferences versus first-principles modelling of the solar interior structure (as traced by the sound speed as a function of depth, as well as quantities such as the helium abundance of the solar envelope and the location of the bottom of the convection zone). The former approach is modelinsensitive; in contrast the latter approach depends the adopted description of the interior opacity, which in turn depends on the assumed solar chemical composition. It has been suggested that an approximately $15 \%$ larger opacity near the base of the convection zone, where oxygen transitions are the largest contributors to the overall opacity together with iron (Mondet et al. 2015), could help to resolve this problem (Christensen-Dalsgaard et al. 2009; Serenelli et al. 2009; Bailey et al. 2015).

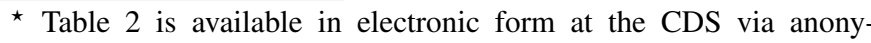
mous ftp to cdsarc.u-strasbg.fr (130.79.128.5) or via http://cdsarc . u-strasbg.fr/viz-bin/qcat?J/A+A/
For the solar photosphere, absorption spectra of the molecules $\mathrm{C}_{2}, \mathrm{CH}, \mathrm{NH}, \mathrm{OH}, \mathrm{CN}$, and ${ }^{12} \mathrm{C}^{16} \mathrm{O}$, offer a promising way to infer the elemental abundances (Lambert 1978; Grevesse et al. 1990, 1991; Asplund et al. 2004, 2005b). The molecules complement the usual atomic indicators of the elemental abundances: namely, the high excitation permitted lines of $\mathrm{C}_{\mathrm{I}}, \mathrm{N}_{\mathrm{I}}$, and $\mathrm{O}_{\mathrm{I}}$ that are typically more sensitive to departures from local thermodynamic equilibrium (LTE; Kiselman 1993; Asplund 2005; Steffen et al. 2015; Amarsi et al. 2020b); as well as the very weak low excitation forbidden lines of $\mathrm{C}_{\mathrm{I}}$ and $\mathrm{O}_{\mathrm{I}}$ which form in LTE but are heavily blended (Caffau et al. 2008; Amarsi et al. 2019). In contrast, the molecular transitions are expected to be less sensitive to departures from LTE (Hinkle \& Lambert 1975; Ayres \& Wiedemann 1989), and there are many unblended features in the solar spectrum to choose from that can be measured with high precision. However, the molecular concentrations are sensitive to the atmospheric structure and the presence of inhomogeneities, especially in high layers near the chromospheric temperature minimum, which makes it essential to base the analysis on time-dependent, three-dimensional (3D) radiative-hydrodynamical models of the photosphere (Asplund et al. 2004, 2005b). Historically, a disadvantage of using molecules came from having to use rather uncertain energy levels and transition probabilities; fortunately, driven in part by their importance in exoplanet modelling (Tennyson \& Yurchenko 2012; Tennyson et al. 2016), data of high accuracy are now available. 
Results from molecules factor into the recommended carbon, nitrogen, and oxygen abundances in the standard solar chemical composition of Asplund et al. (2009). However, there have been a number of developments since that work, that make a new analysis worthwhile. First, improved data have recently become available for all of these molecules: primarily transition probabilities (Brooke et al. 2013, 2014a,b, 2015, 2016; Masseron et al. 2014; Li et al. 2015); and also molecular partition functions and equilibrium constants (Barklem \& Collet 2016). Secondly, a number of accurate, complementary results have become available, and it is important to verify that consistent results are obtained. These include 3D non-LTE elemental abundances based on $\mathrm{C}_{\mathrm{I}}$ (Amarsi et al. 2019; Li et al. 2021), N I (Amarsi et al. 2020a), and O I (Amarsi et al. 2018). Lastly, the 3D model atmosphere has been updated, as summarised in Table 1 of Asplund et al. (2021); in particular, the newer model was constructed with the composition of (Asplund et al. 2009), rather than the more metal-poor composition of (Asplund et al. 2005a).

Owing to these developments, we here present a selection and analysis of the best molecular diagnostics in the solar spectrum. Preliminary results of the present study were already incorporated into the new solar chemical composition of Asplund et al. (2021). We first present the selection of lines and their equivalent widths, and review how the 3D LTE theoretical spectra were calculated (Sect. 2). We describe the analysis method and present the final results (Sect. 3), and discuss them (Sect. 4), before presenting our conclusions (Sect. 5).

\section{Method}

\subsection{Observational data and line selection}

Our analysis is based on the solar disk-centre intensity spectrum. Different standard high-resolution solar atlases were used for different lines, depending on their wavelengths. For lines in the optical and near-infrared, the ground-based Liège (Delbouille et al. 1973; Delbouille et al. 1981; Delbouille \& Roland 1995) and Hamburg (Neckel \& Labs 1984; Neckel 1999) atlases (see Doerr et al. 2016 for an informative review and comparison of these atlases and others in terms of e.g. spectral resolution, telluric absorption and continuum placement) formed the observational basis. For lines in the infrared, the space-based ATMOS (Abrams et al. 1996) and ACE (Hase et al. 2010) atlases were used.

In general, the equivalent widths of selected lines were determined separately in both atlases, by identifying the continuum from local maxima within a few tenths of a nanometre on either side of the central depression, and subsequently measuring the area under the continuum by hand using a planimeter. The mean equivalent widths from the two atlases were adopted; these are typically in excellent agreement, but can vary by up to around $5 \%$ for problematic lines: for instance, the $\mathrm{C}_{2}$ lines, which are typically weak and sit in a crowded region of the optical spectrum, which makes the continuum difficult to place and profile shapes difficult to trace. The first overtone $\mathrm{OH}$ lines are an exception, this analysis being based on the equivalent widths of Meléndez (2004), measured in the atlas of Wallace \& Livingston (2003). To give some feeling for the spectral features analysed in this work, we show a $\mathrm{CH}$ optical line and $\mathrm{OH}$ infrared line in Fig. 1.

In Table 1 we summarise the different groups of lines considered, and the number of lines in each group. We present the entire list of line parameters and their measured equivalent widths in Table 2. These lines were chosen for being free of blends and sufficiently strong to be measured reliably, while remaining on or close to the linear part of the curve of growth, to enable the elemental abundances to be inferred with high precision. The wavelengths, energies, and transition probabilities were adopted from Brooke et al. $(2013,2014 b, 2015,2016)$ for $\mathrm{C}_{2}, \mathrm{CN}, \mathrm{NH}$, and $\mathrm{OH}$ respectively; from Masseron et al. (2014) for $\mathrm{CH}$; and from $\mathrm{Li}$ et al. (2015) for ${ }^{12} \mathrm{C}^{16} \mathrm{O}$.

Many more lines were also considered, but not retained in the final analysis. Most notably, a further 463 lines of 14 other bands in the red system of $\mathrm{CN}$ were studied and initially kept (Asplund et al. 2021). The analysis of these lines was based on equivalent widths that were measured automatically over 20 years ago for the purposes of line identification (A. J. Sauval, priv. comm.). The mean results from these various bands were found to agree with those from the $(0-0)$ band to within 0.05 dex. However, the dispersions of the results are two to three times larger because of random uncertainties in the equivalent widths. For this reason, these 463 transitions were not retained in the final analysis. In addition, several lines in the $\mathrm{NH} \mathrm{A-X,} \mathrm{OH} \mathrm{A-X,} \mathrm{and} \mathrm{CN}$ $\mathrm{B}-\mathrm{X}$ electronic systems were also considered. Their lines fall in the near UV, around $340 \mathrm{~nm}, 320 \mathrm{~nm}$, and $390 \mathrm{~nm}$, respectively. There is a high density of spectral lines in these spectral regions, making the precise measurement of equivalent widths extremely difficult because of the contamination by other species and because of the difficulty to find the real continuum. For these reasons, these molecular transitions were ultimately not included.

\subsection{Model atmospheres}

In Fig. 2 we illustrate the temperature structure as a function of Rosseland optical depth of the model atmospheres used in this study. The analysis was based on a 3D radiative-hydrodynamic model of the solar photosphere computed with the STAGGER code (Collet et al. 2011; Magic et al. 2013). The mean effective temperature of the entire sequence (as well as of the sample of snapshots; Sect. 2.3) is $5773 \mathrm{~K}$, with a standard deviation of $16 \mathrm{~K}$, in excellent agreement with the nominal solar value of $5772 \mathrm{~K}$ (Prša et al. 2016). The model is the same as that used already in the 3D non-LTE analyses of O I lines (Amarsi et al. 2018), where it is discussed in more detail; as well as of $\mathrm{C}_{\mathrm{I}}$ and $\mathrm{N}_{\mathrm{I}}$ lines (Amarsi et al. 2019; Li et al. 2021; Amarsi et al. 2020a). It was also used in the 3D non-LTE analyses of the sodium, magnesium, potassium, calcium, and iron abundances, presented in Asplund et al. (2021). It is an updated version of the model used in the solar analyses of Asplund et al. (2009) as well as of Scott et al. (2015b,a) and Grevesse et al. (2015), using the latest version of the STAGGER code as described by Collet et al. (2018), with an updated chemical composition (Asplund et al. 2009); Table 1 of Asplund et al. (2021) summarises some of the differences with the older generations of the 3D solar model. Test calculations on an older version of the current 3D model suggest that the differences in inferred abundance may be roughly around 0.01 dex for most of the molecular indicators considered here.

For comparison purposes, as well as to estimate systematic errors, several 1D hydrostatic models were also used in this study. These are namely: a temporal- and horizontal-average (on surfaces of equal Rosseland mean optical depth) of the full 3D model (Sect. 2.1.2 of Amarsi et al. 2018), hereafter referred to as the $\langle 3 \mathrm{D}\rangle$ model; a theoretical ATMO model (Appendix A of Magic et al. 2013), that is calculated with the same radiative transfer scheme and input physics as the 3D STAGGER model; the solar model from the theoretical MARCS grid (Gustafsson et al. 2008), which is copiously used in stellar abundance analyses; and the 

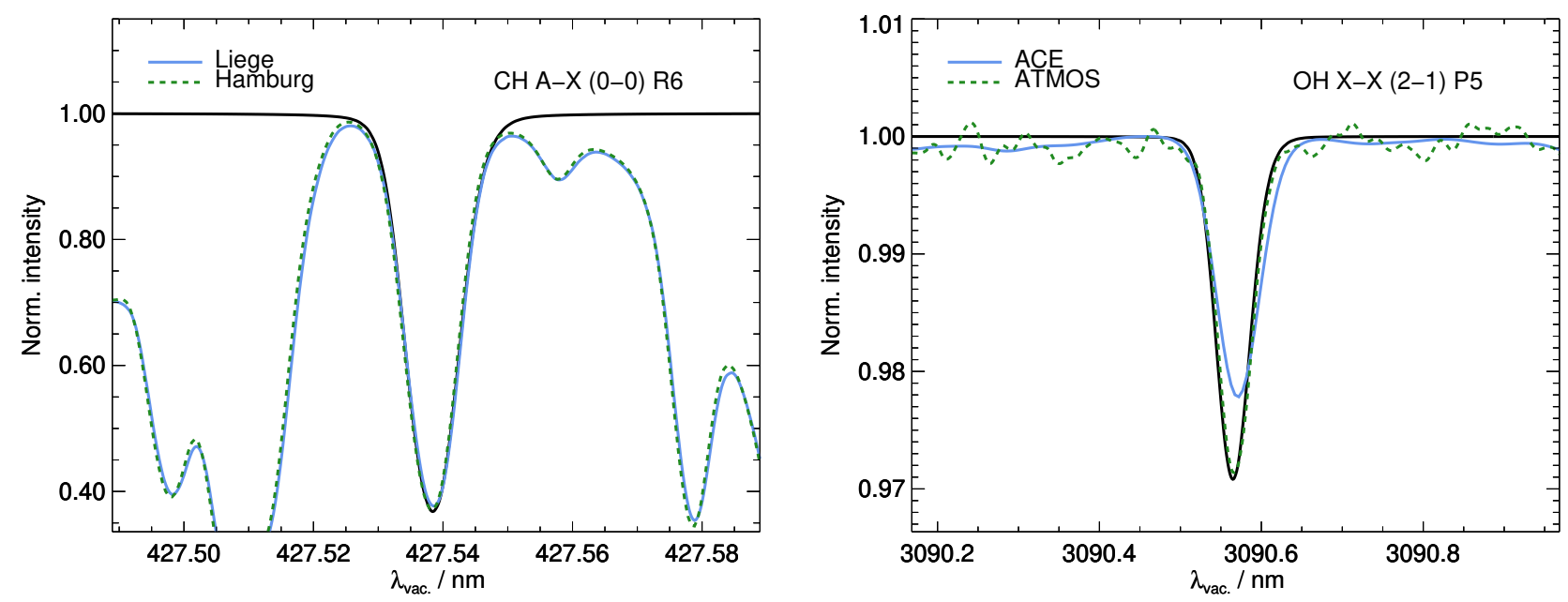

Fig. 1. Illustrative example optical and infrared spectra from the $3 \mathrm{D}$ model compared to different solar atlases. The theoretical line strengths have not been fit to the data; rather, the input abundance is based on interpolating the theoretical equivalent-width to the mean one measured in both atlases. No instrumental broadening has been applied, and thus in the infrared the ACE profiles are seen to be broader and shallower than both the ATMOS and theoretical ones.

Table 1. Groups of indicators used in the present analysis.

\begin{tabular}{ccccccc}
\hline \hline Species & System & $|\Delta v|$ & Bands & $N_{\text {line }}$ & $\lambda_{\text {vac. }} / \mathrm{nm}$ & Param. \\
\hline \hline $\mathrm{C}_{2}$ & Swan & 0 & $(0-0),(1-1)$ & 32 & $494-516$ & \\
$\mathrm{C}_{2}$ & Swan & 1 & $(0-1),(1-0),(1-2)$ & 7 & $473-562$ & \\
$\mathrm{CH}$ & $\mathrm{A}-\mathrm{X}$ & 0 & $(0-0),(1-1)$ & 6 & $425-436$ & $\log \epsilon_{\mathrm{C}}$ \\
$\mathrm{CH}$ & $\mathrm{X}-\mathrm{X}$ & 1 & $(1-0),(2-1),(3-2)$ & 48 & $3295-3795$ & \\
${ }^{12} \mathrm{C}^{16} \mathrm{O}$ & $\mathrm{X}-\mathrm{X}$ & 1 & $(1-0),(2-1),(3-2),(4-3),(5-4)$ & 28 & $4297-6329$ & \\
${ }^{12} \mathrm{C}^{16} \mathrm{O}$ & $\mathrm{X}-\mathrm{X}$ & 2 & $(2-0),(3-1)$ & 52 & $2295-2591$ & \\
\hline $\mathrm{NH}$ & $\mathrm{X}-\mathrm{X}$ & 0 & $(0-0),(1-1)$ & 13 & $11311-15023$ & \\
$\mathrm{NH}$ & $\mathrm{X}-\mathrm{X}$ & 1 & $(1-0),(2-1),(3-2),(4-3)$ & 18 & $2891-3445$ & $\log \epsilon_{\mathrm{N}}$ \\
$\mathrm{CN}$ & $\mathrm{A}-\mathrm{X}$ & 0 & $(0-0)$ & 59 & $1088-1321$ & \\
\hline $\mathrm{OH}$ & $\mathrm{X}-\mathrm{X}$ & 0 & $(0-0),(1-1),(2-2)$ & 84 & $9389-12280$ & \\
$\mathrm{OH}$ & $\mathrm{X}-\mathrm{X}$ & 1 & $(1-0),(2-1)$ & 46 & $2970-3813$ & $\log \epsilon_{\mathrm{O}}$ \\
$\mathrm{OH}$ & $\mathrm{X}-\mathrm{X}$ & 2 & $(2-0),(3-1),(4-2)$ & 15 & $1528-1762$ & \\
\hline \hline
\end{tabular}

Notes. Final column specifies the free parameter that is adjusted so as to match the theoretical equivalent widths to the observed ones, in the analysis that follows. $\mathrm{C}_{2}$ Swan, $\mathrm{CH}$ A-X and CN A-X are electronic transitions. NH X-X $|\Delta v|=0$ and OH X-X $|\Delta v|=0$ are rotational (or pure-rotation) transitions. The $\mathrm{CH} \mathrm{X}-\mathrm{X},{ }^{12} \mathrm{C}^{16} \mathrm{O} X-\mathrm{X}, \mathrm{NH} \mathrm{X}-\mathrm{X}|\Delta v|=1, \mathrm{OH} \mathrm{X}-\mathrm{X}|\Delta v| \geq 1$ are rovibrational (or vibration-rotation) transitions.

semi-empirical model of Holweger \& Müller (1974), hereafter the HM74 model. The latter model was originally constructed so as to reproduce a number of key observational diagnostics: the centre-to-limb variation of the continuum as well as the wings of Fraunhofer lines to probe the $T(\tau)$ relation of the thicker layers of the photosphere; and the centre-to-limb variations of the central intensities of around 900 atomic and ionic lines to probe the thinner layers (Holweger 1967). We still refer to this last model because it has been used for many decades in a very large number of works, but it should be noted that its temperature structure may be slightly too warm in the layers where typical atomic lines used for abundance purposes are formed (Asplund et al. 2009; see also Sect. 4.2).

\subsection{Line formation calculations}

The vertical intensities emergent from the 3D model, and the four 1D models, were generated using the 3D LTE radiative transfer code SCATE (Hayek et al. 2011). The atomic and molecular partition functions and equilibrium constants were updated to use the data from Barklem \& Collet (2016). When computing the chemical equilibrium, SCATE includes $\mathrm{H}^{-}$, atoms and ions up to and including the third ionisation stage (e.g. Fe III), as well as 12 molecules of hydrogen, carbon, nitrogen, and oxygen: $\mathrm{H}_{2}$, $\mathrm{H}_{2}^{+} ; \mathrm{C}_{2}, \mathrm{~N}_{2}, \mathrm{O}_{2} ; \mathrm{CH}, \mathrm{NH}, \mathrm{OH} ; \mathrm{CN}, \mathrm{CO}, \mathrm{NO}$; and $\mathrm{H}_{2} \mathrm{O}$.

For the $3 \mathrm{D}$ model, the calculations were performed on 52 snapshots each roughly twenty minutes of solar time apart, to adequately sample the solar granulation. Compared to the original simulation, the horizontal resolution was reduced by a factor of $3 \times 3$, from $240^{2}$ grid points to $80^{2}$ grid points, while the 
Table 2. Lines used in the present analysis.

\begin{tabular}{|c|c|c|c|c|c|c|c|c|c|c|c|c|c|}
\hline Species & System & $|\Delta v|$ & Band & $\lambda_{\text {vac. }} / \mathrm{nm}$ & $E_{\text {low }} / \mathrm{eV}$ & $\log g f$ & $W / \mathrm{pm}$ & Param. & $3 \mathrm{D}$ & $\langle 3 \mathrm{D}\rangle$ & ATMO & MARCS & HM74 \\
\hline $\mathrm{C}_{2}$ & Swan & 0 & $(0-0)$ & 493.812 & 1.112 & 0.843 & 0.530 & $\log \epsilon_{\mathrm{C}}$ & 8.401 & 8.415 & 8.380 & 8.376 & 8.459 \\
\hline $\mathrm{C}_{2}$ & Swan & 0 & $(0-0)$ & 495.281 & 1.029 & 0.651 & 0.500 & $\log \epsilon_{\mathrm{C}}$ & 8.442 & 8.457 & 8.422 & 8.417 & 8.501 \\
\hline$\cdots$ & $\cdots$ & $\cdots$ & $\cdots$ & $\cdots$ & $\cdots$ & $\cdots$ & $\cdots$ & $\cdots$ & $\cdots$ & $\cdots$ & $\cdots$ & $\cdots$ & $\cdots$ \\
\hline $\mathrm{CH}$ & A-X & 0 & $(1-1)$ & 425.420 & 0.523 & -1.506 & 3.700 & $\log \epsilon_{\mathrm{C}}$ & 8.418 & 8.433 & 8.368 & 8.357 & 8.512 \\
\hline $\mathrm{CH}$ & A-X & 0 & $(1-1)$ & 425.441 & 0.523 & -1.471 & 4.000 & $\log \epsilon_{\mathrm{C}}$ & 8.422 & 8.437 & 8.372 & 8.360 & 8.517 \\
\hline$\ldots$ & .. & $\ldots$ & $\cdots$ & & $\cdots$ & $\cdots$ & $\cdots$ & & . & $\ldots$ & $\cdots$ & $\ldots$ & $\cdots$ \\
\hline $\mathrm{CH}$ & $X-X$ & 1 & $(1-0)$ & 3294.615 & 0.773 & -2.600 & 1.590 & $\log \epsilon_{\mathrm{C}}$ & 8.455 & 8.485 & 8.424 & 8.418 & 8.555 \\
\hline $\mathrm{CH}$ & $X-X$ & 1 & $(1-0)$ & 3303.095 & 0.915 & -2.523 & 1.460 & $\log \epsilon_{\mathrm{C}}$ & 8.479 & 8.508 & 8.446 & 8.441 & 8.576 \\
\hline$\ldots$ & $\ldots$ & $\ldots$ & $\ldots$ & $\ldots$ & $\ldots$ & $\ldots$ & $\ldots$ & $\ldots$ & $\ldots$ & $\ldots$ & $\ldots$ & $\ldots$ & $\ldots$ \\
\hline${ }^{12} \mathrm{C}^{16} \mathrm{O}$ & $X-X$ & 1 & $(1-0)$ & 4297.175 & 2.242 & -2.876 & 8.240 & $\log \epsilon_{\mathrm{C}}$ & 8.482 & 8.580 & 8.550 & 8.540 & 8.623 \\
\hline${ }^{12} \mathrm{C}^{16} \mathrm{O}$ & $X-X$ & 1 & $(1-0)$ & 4297.866 & 2.286 & -2.872 & 7.460 & $\log \epsilon_{\mathrm{C}}$ & 8.462 & 8.561 & 8.530 & 8.522 & 8.605 \\
\hline$\cdots$ & $\cdots$ & $\cdots$ & $\cdots$ & .. & $\cdots$ & $\cdots$ & $\cdots$ & $\cdots$ & .. & $\cdots$ & $\cdots$ & $\cdots$ & $\cdots$ \\
\hline $\mathrm{NH}$ & $X-X$ & 0 & $(0-0)$ & 11311.232 & 2.138 & -1.557 & 0.243 & $\log \epsilon_{\mathrm{N}}$ & 7.898 & 8.002 & 8.015 & 7.997 & 8.088 \\
\hline $\mathrm{NH}$ & $X-X$ & 0 & $(0-0)$ & 11471.850 & 2.027 & -1.570 & 0.316 & $\log \epsilon_{\mathrm{N}}$ & 7.913 & 8.012 & 8.035 & 8.003 & 8.039 \\
\hline$\cdots$ & A-X & 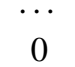 & $(0-0)$ & 1087. & 0.056 & -2.601 & 0.136 & 10 & 7.870 & 8.000 & 7.964 & 7.957 & 8.051 \\
\hline $\mathrm{CN}$ & A-X & 0 & $(0-0)$ & 1087.548 & 0.089 & -2.630 & 0.112 & $\log \epsilon_{\mathrm{N}}$ & 7.848 & 7.965 & 7.944 & 7.932 & 8.027 \\
\hline$\cdots$ & $\cdots$ & $\cdots$ & $\cdots$ & $\cdots$ & $\cdots$ & $\cdots$ & $\cdots$ & $\cdots$ & $\cdots$ & $\ldots$ & $\ldots$ & $\ldots$ & $\ldots$ \\
\hline $\mathrm{OH}$ & $X-X$ & 0 & $(0-0)$ & 9389.310 & 3.070 & -1.227 & 1.798 & $\log \epsilon_{\mathrm{O}}$ & 8.718 & 8.779 & 8.770 & 8.761 & 8.834 \\
\hline $\mathrm{OH}$ & $\mathrm{X}-\mathrm{X}$ & 0 & $(0-0)$ & 9391.329 & 3.071 & -1.239 & 1.817 & $\log \epsilon_{\mathrm{O}}$ & 8.734 & 8.797 & 8.789 & 8.784 & 8.851 \\
\hline$\ldots$ & .. & $\ldots$ & $\cdots$ & & .. & $\ldots$ & $\cdots$ & & $\ldots$ & $\ldots$ & $\ldots$ & $\cdots$ & $\ldots$ \\
\hline
\end{tabular}

Notes. Columns 1 to 7 show the line information, column 8 shows the equivalent width measured in the solar disc-centre intensity, and column 9 specifies the free parameter that is adjusted so as to match the theoretical equivalent widths to the observed ones. Final columns show the elemental abundances inferred from the different models. Blended transitions within the same branch were in some cases combined together. The NH lines are all in the $\mathrm{R}$ branch with three unresolved components. The listed transition probabilities are for the middle component, and the measured equivalent width has been reduced by a factor of three. Two lines of each system are shown; the full table is available online.

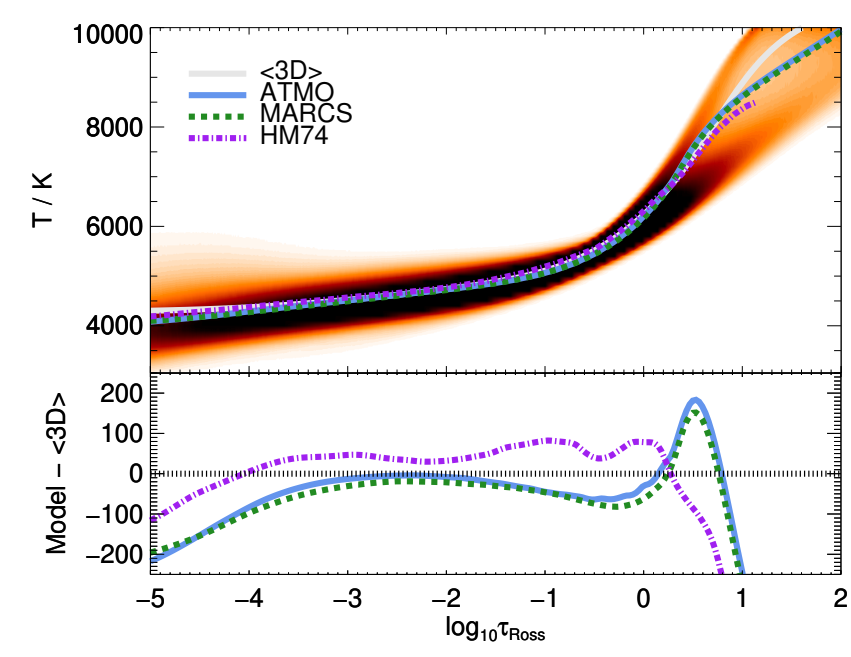

Fig. 2. Distribution of temperature and Rosseland mean vertical optical depth for the 3D radiative-hydrodynamical model atmosphere, with darker colours indicating larger densities of grid points. The structures of the 1D model atmospheres used in this work are overplotted. Bottom panel shows the difference in temperature of the 1D models with respect to the horizontally and temporally averaged $\langle 3 \mathrm{D}\rangle$ model.

vertical resolution was enhanced by a factor of 2 near the op- tical surface. The intensities were calculated without any extra microturbulent broadening, these non-thermal effects being naturally accounted for due to the velocity and temperature inhomogeneities in the 3D model itself (Asplund et al. 2000; Nordlund et al. 2009). The calculations on the $1 \mathrm{D}$ models were performed in almost the same manner. The main difference is that in the latter case, a depth-independent microturbulence was adopted, $\xi_{\text {mic }}=1.0 \mathrm{~km} \mathrm{~s}^{-1}$ (Holweger et al. 1978).

The abundances of all three of carbon, nitrogen, and oxygen can influence the theoretical intensity depressions of all the lines in Table 2, through changes to the equation of state and molecular balance. Therefore, for all of the model atmospheres, all line intensities were calculated for a three-dimensional rectilinear grid of carbon, nitrogen, and oxygen abundances. The abundances were taken to be between -0.2 dex to +0.2 dex in steps of 0.1 dex about central values of $8.43,7.83$, and 8.69 , respectively (Asplund et al. 2009), resulting in a total of $5 \times 5 \times 5$ different chemical compositions.

The subsequent analysis is based on comparing theoretical equivalent widths onto the observational data (Sect. 2.1). The theoretical equivalent widths were interpolated in the threedimensional abundance space using cubic splines. For all of the lines predicted by the 3D model, the inferred elemental abundances all fell within the grids stipulated above. However, for the 1D models, there were some deviations beyond the edges of these grids, stipulated above. Since the 1D results are not a pri- 
ority of the present study, linear extrapolations were applied in those cases.

\section{Abundance analysis and results}

\subsection{Preliminary comments}

Combining the individual lines in Table 2 into a final estimate of the elemental abundances is not a straightforward task. One can see that, by taking an unweighted mean of all of the lines would bias the result towards those groups or molecules in Table 1 having the largest number of analysed transitions. This is undesirable because lines of different molecules, electronic systems, and $|\Delta v|$ are susceptible to different systematics, and the analysis should take advantage of this. This is attempted here, as described in the remainder of Sect. 3.

\subsection{Iterative procedure}

The analysis proceeds in an iterative fashion, because the inferred chemical composition, and its uncertainty, has an effect on the theoretical equivalent widths and thus the inferred abundances. In the initialisation step, a guess was made on the modeldependent abundances of carbon, nitrogen, and oxygen, as well as on their uncertainties. The choice of initial guess was not critical, and varying it across the extent of the calculated grid did not alter the final converged results. For a given line, two of the three abundances were fixed; the free parameter is specified in Table 1 . For $\mathrm{CN}$ and ${ }^{12} \mathrm{C}^{16} \mathrm{O}$, the free parameter is the less abundant element: nitrogen and carbon respectively.

In the first step, the lines were grouped according to the rows in Table 1. The assumption is that lines of the same molecule, in the same electronic system, with the same $|\Delta v|$ have similar sensitivities to systematic errors implicit in the model atmospheres and molecular data, and should therefore be grouped together. For each model, unweighted mean values, $\log \epsilon$ (model; group), were determined by summing over all lines in a given group. The squared uncertainties were calculated as follows:

$$
\begin{aligned}
\sigma^{2}(\text { model } ; \text { group })= & \sigma^{2}(\text { group } ; \text { atmos }) \\
& +\sigma^{2}(\text { model } ; \text { group } ; \text { trend }) \\
& +\sigma^{2}(\text { model } ; \text { group } ; \text { comp }) \\
& +\sigma^{2}(\text { model } ; \text { group } \text { stat })
\end{aligned}
$$

The first term in Eq. 1 accounts for the sensitivity of the group to the model atmosphere. Here, this was estimated as the standard error in the means of the results from the four different modelling paradigms: 3D, $\langle 3 \mathrm{D}\rangle, 1 \mathrm{D}$ theoretical ATMO, and $1 \mathrm{D}$ semi-empirical HM74; it is therefore the same for all models. The second term in Eq. 1 probes further shortcomings that may be present in all model atmospheres, for example systematic biases in the equivalent width measurements or molecular data. This was evaluated by fitting linear trends as functions of different line parameters (namely, wavelength, excitation potential, and logarithmic reduced equivalent width): the most severe trend was identified, and half the difference between the highest and lowest point of the fit was evaluated. The third term in Eq. 1 accounts for the uncertainty in the two fixed background abundances propagated onto the fitted third abundance, omitting the covariance terms. The final term in Eq. 1 gives the statistical uncertainty. It was evaluated as the sample standard deviation about the fitted trend (noting that there are $N_{\text {line }}-2$ degrees of freedom), divided by $\sqrt{N_{\text {line }}}$. Any random errors in the equivalent widths and transition probabilities were assumed to be implicit in this quantity.

In the second step, the results for different groups were collapsed so as to obtain mean values for each molecule and each model. This was achieved via a weighted mean. The weights were calculated for each model and group, via the uncertainties evaluated using Eq. 1:

$$
\mathrm{w}(\text { model } ; \text { group })=\frac{\sigma^{-2}(\text { model } ; \text { group })}{\sum \sigma^{-2}(\text { model } ; \text { group })} .
$$

Here, the summation in the denominator ensure that the weights for all of the groups of a given molecule sum up to unity (i.e. that they are normalised). The results for different groups, $\log \epsilon$ (model; group), were thus weighted using Eq. 2 and added together so as to determine the weighted mean, $\log \epsilon$ (model; molecule).

To calculate the uncertainty in this weighted mean, the four uncertainty components appearing in Eq. 1 (atmospheric, trend, compositional, and statistical, respectively) were individually propagated forward using the following general formula:

$$
\left\langle\sigma^{2}\right\rangle=\sum_{i}^{n} w_{i}^{2} \sigma_{i}^{2}+\sum_{i}^{n} \sum_{j \neq i}^{n} w_{i} w_{j} \rho_{i, j} \sigma_{i} \sigma_{j} .
$$

For example, for a given model and molecule, the quantity $\sigma$ (model; molecule; trend) was determined by setting $w_{i}$ and $w_{j}$ to w (model; group), and $\sigma_{i}$ and $\sigma_{j}$ to $\sigma$ (model; group; trend), and summing over the different groups as denoted by the indices $i$ and $j$ in Eq. 3. The statistical and trend uncertainties were assumed to be uncorrelated, and hence the correlation coefficients $\rho_{i, j}$ were taken to be zero. In contrast both the atmospheric and the compositional uncertainties were assumed to be systematic and thus perfectly correlated between different groups of the same molecule, and therefore $\rho_{i, j}=1$. For a given model and molecule, the uncertainty, $\sigma$ (model; molecule), was then determined by adding these four propagated components together in quadrature.

In the third step, the results for different molecules were combined into final determinations of the elemental abundances. Similarly to the previous step, these were calculated through a weighted mean, summing over all molecules of a given element. The four uncertainty components were propagated forward again using Eq. 3, this time summing over all molecules of a particular element, rather than all groups of a particular molecule. The final uncertainties in the elemental abundances were given by adding the four components in quadrature.

With a new estimate of the elemental abundances in hand, as well as an improved estimate of the composition error, $\sigma$ (model; group; comp.), appearing in Eq. 1, the next iteration cycle was initiated. This proceeded until the elemental abundances had converged to five decimal places.

One last note to make is that the line-by-line abundances were adjusted to take into account that the line formation calculations were performed under the assumption that all $\mathrm{CO}$ is in the major isotopologue, ${ }^{12} \mathrm{C}^{16} \mathrm{O}$. Using for example Eq. 1 of Ayres et al. (2013), and the isotopic ratios advocated in Asplund et al. (2021), this amounted to an increase in the carbon abundances inferred from the ${ }^{12} \mathrm{C}^{16} \mathrm{O}$ lines by only $0.006 \mathrm{dex}$.

\subsection{Advocated elemental abundances}

We provide the converged line-by-line values in the final five columns of Table 2, corresponding to the five different models 
Table 3. Inferred elemental abundances and uncertainties.

\begin{tabular}{|c|c|c|c|c|c|c|c|c|c|c|c|c|}
\hline Species & System & $|\Delta v|$ & $3 \mathrm{D}$ & $\langle 3 \mathrm{D}\rangle$ & ATMO & MARCS & HM74 & $\sigma($ atmos. $)$ & $\sigma($ trend $)$ & $\sigma$ (comp.) & $\sigma$ (stat.) & $\sigma$ \\
\hline \multicolumn{13}{|c|}{ Carbon } \\
\hline $\mathrm{C}_{2}$ & Swan & 0 & 8.455 & 8.473 & 8.438 & 8.433 & 8.520 & 0.018 & 0.003 & 0.006 & 0.005 & 0.020 \\
\hline $\mathrm{C}_{2}$ & Swan & 1 & 8.474 & 8.494 & 8.459 & 8.454 & 8.540 & 0.018 & 0.004 & 0.006 & 0.010 & 0.022 \\
\hline \multicolumn{3}{|c|}{$\left\langle\mathrm{C}_{2}\right\rangle$} & 8.464 & 8.483 & 8.448 & 8.443 & 8.530 & 0.018 & 0.003 & 0.006 & 0.005 & 0.020 \\
\hline $\mathrm{CH}$ & A-X & 0 & 8.459 & 8.471 & 8.404 & 8.393 & 8.555 & 0.031 & 0.015 & 0.005 & 0.011 & 0.037 \\
\hline $\mathrm{CH}$ & $X-X$ & 1 & 8.470 & 8.503 & 8.444 & 8.438 & 8.573 & 0.028 & 0.017 & 0.006 & 0.003 & 0.033 \\
\hline \multicolumn{3}{|c|}{$\langle\mathrm{CH}\rangle$} & 8.465 & 8.489 & 8.427 & 8.420 & 8.565 & 0.029 & 0.011 & 0.006 & 0.005 & 0.032 \\
\hline${ }^{12} \mathrm{C}^{16} \mathrm{O}$ & $X-X$ & 1 & 8.487 & 8.653 & 8.617 & 8.606 & 8.683 & 0.043 & 0.021 & 0.031 & 0.004 & 0.057 \\
\hline${ }^{12} \mathrm{C}^{16} \mathrm{O}$ & $X-X$ & 2 & 8.467 & 8.681 & 8.623 & 8.609 & 8.743 & 0.059 & 0.016 & 0.029 & 0.001 & 0.068 \\
\hline \multicolumn{3}{|c|}{$\left\langle{ }^{12} \mathrm{C}^{16} \mathrm{O}\right\rangle$} & 8.479 & 8.673 & 8.621 & 8.608 & 8.723 & 0.050 & 0.014 & 0.030 & 0.002 & 0.060 \\
\hline \multirow{2}{*}{\multicolumn{3}{|c|}{ Final }} & 8.47 & 8.49 & 8.45 & 8.45 & 8.55 & 0.02 & 0.00 & 0.01 & 0.00 & 0.02 \\
\hline \multicolumn{12}{|c|}{ Nitrogen } & \\
\hline $\mathrm{NH}$ & $X-X$ & 0 & 7.881 & 8.032 & 8.038 & 8.021 & 8.079 & 0.043 & 0.036 & 0.000 & 0.006 & 0.057 \\
\hline $\mathrm{NH}$ & $\mathrm{X}-\mathrm{X}$ & 1 & 7.921 & 7.988 & 7.922 & 7.914 & 8.056 & 0.032 & 0.008 & 0.001 & 0.005 & 0.033 \\
\hline \multicolumn{3}{|c|}{$\langle\mathrm{NH}\rangle$} & 7.911 & 8.002 & 7.960 & 7.951 & 8.065 & 0.035 & 0.011 & 0.000 & 0.004 & 0.037 \\
\hline $\mathrm{CN}$ & A-X & 0 & 7.867 & 7.964 & 7.933 & 7.927 & 8.016 & 0.031 & 0.008 & 0.030 & 0.003 & 0.044 \\
\hline \multicolumn{3}{|c|}{$\langle\mathrm{CN}\rangle$} & 7.867 & 7.964 & 7.933 & 7.927 & 8.016 & 0.031 & 0.008 & 0.030 & 0.003 & 0.044 \\
\hline \multicolumn{3}{|c|}{ Final } & 7.89 & 7.99 & 7.95 & 7.94 & 8.04 & 0.03 & 0.01 & 0.01 & 0.00 & 0.04 \\
\hline \multicolumn{13}{|c|}{ Oxygen } \\
\hline $\mathrm{OH}$ & $\mathrm{X}-\mathrm{X}$ & 0 & 8.690 & 8.792 & 8.795 & 8.782 & 8.837 & 0.031 & 0.041 & 0.005 & 0.001 & 0.052 \\
\hline $\mathrm{OH}$ & $X-X$ & 1 & 8.707 & 8.800 & 8.737 & 8.728 & 8.884 & 0.039 & 0.008 & 0.004 & 0.002 & 0.040 \\
\hline $\mathrm{OH}$ & $\mathrm{X}-\mathrm{X}$ & 2 & 8.690 & 8.756 & 8.685 & 8.673 & 8.848 & 0.038 & 0.066 & 0.003 & 0.012 & 0.077 \\
\hline \multicolumn{3}{|c|}{$\langle\mathrm{OH}\rangle$} & 8.699 & 8.791 & 8.756 & 8.751 & 8.852 & 0.036 & 0.017 & 0.004 & 0.002 & 0.040 \\
\hline \multicolumn{3}{|c|}{ Final } & 8.70 & 8.79 & 8.76 & 8.75 & 8.85 & 0.04 & 0.02 & 0.00 & 0.00 & 0.04 \\
\hline
\end{tabular}

Notes. Abundances are shown for different groups and species, for all of the models considered in the present analysis. Also shown is the breakdown of uncertainties for the 3D model, with the overall uncertainty given in the final column.

considered here. In Table 3, we show the abundances inferred from the different groups, the collapsed results for the different molecules, and our final advocated elemental abundances, for each of the different models. This table also shows the full breakdown of uncertainties, albeit only for the 3D model, because in practice they are dominated by the atmospheric uncertainty, so are similar for the other models.

In Table 3, the final advocated abundances as inferred from the molecules are $\log \epsilon_{\mathrm{C}}=8.47 \pm 0.02, \log \epsilon_{\mathrm{N}}=7.89 \pm 0.04$, and $\log \epsilon_{\mathrm{O}}=8.70 \pm 0.04$. The stipulated uncertainties are dominated by the atmospheric uncertainty. These were taken to be correlated ( $\rho_{i, j}=1$ in Eq. 3 ), which is why the individual groups and molecules typically agree with the final values to better than these stated precisions of 0.02 to $0.04 \mathrm{dex}$. The largest deviation was found for the $\mathrm{NH}|\Delta v|=1$ group, for which the nitrogen abundance is found to be 0.03 dex larger than the final result.

\section{Discussion}

\subsection{Line-by-line results in the $3 D$ model}

Figs. 3, 4, and 5 illustrate the line-by-line abundances for the 3D model, for carbon, nitrogen, and oxygen respectively. The individual panels show the results for different molecules, plotted against excitation potential and reduced equivalent width. In addition, for each group of indicators, the difference between the average results from the $1 \mathrm{D}$ models and from the $3 \mathrm{D}$ model are shown in Fig. 6.

The line-by-line standard deviations, i.e. the scatter about the mean trends in Figs. 3, 4, and 5, are found to be around 0.02 dex. The lowest scatters are found for the ${ }^{12} \mathrm{C}^{16} \mathrm{O}|\Delta v|=2$ and $\mathrm{OH}|\Delta v|=1$ groups $(0.01 \mathrm{dex})$. This scatter can be partially explained by random measurement errors, that can reach of the order $2.5 \%$ or 0.01 dex (Sect. 2.1); random uncertainties in the adopted transition probabilities likely also contribute. An anomalously large scatter is found for the $\mathrm{OH}|\Delta v|=2$ group $(0.05 \mathrm{dex})$. This group displays a severe trend with reduced equivalent width, which likely reflects particular difficul- 

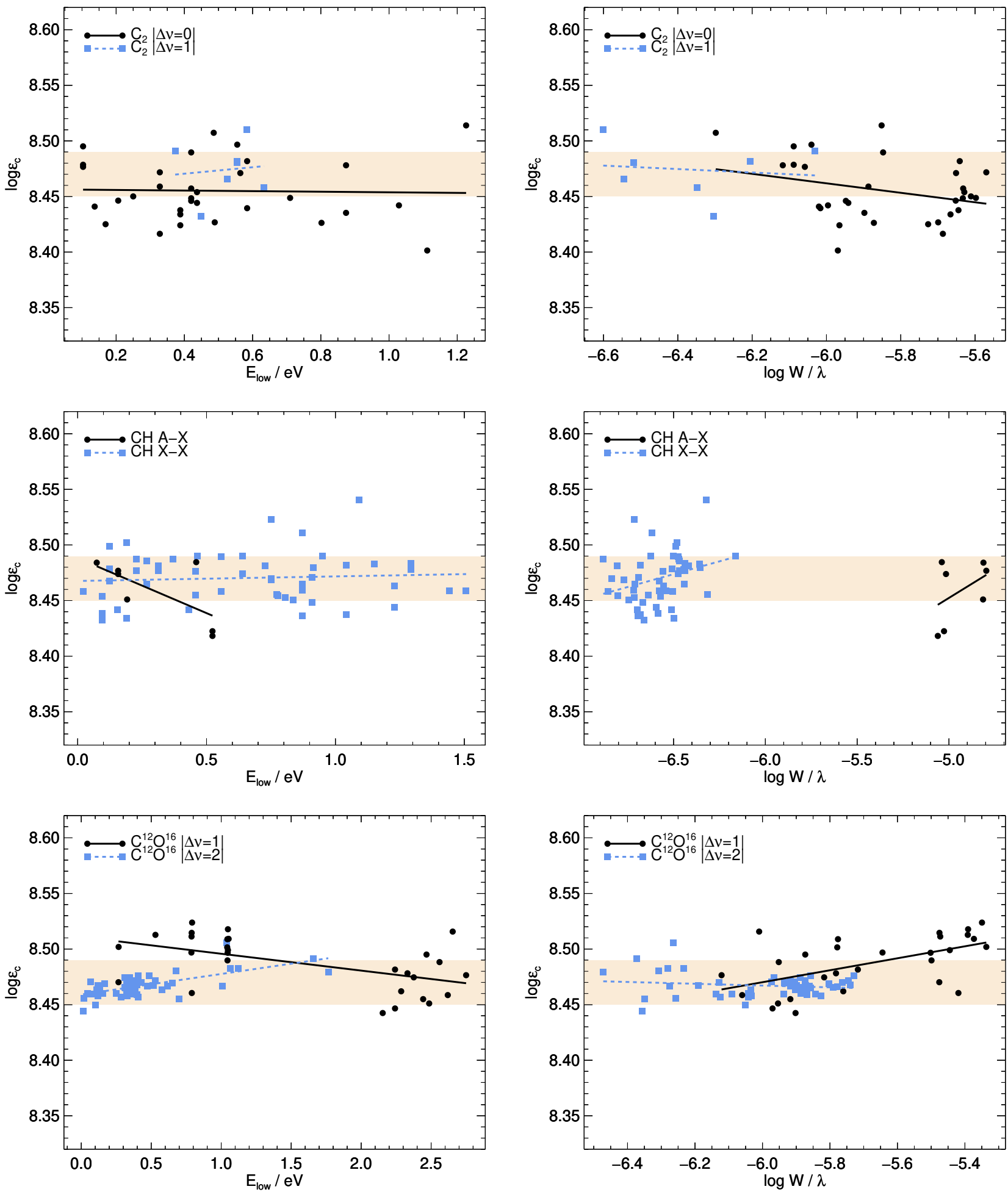

Fig. 3. Carbon abundances inferred from lines of $\mathrm{C}_{2}, \mathrm{CH}$, and ${ }^{12} \mathrm{C}^{16} \mathrm{O}$ (rows), against excitation potential and reduced equivalent width (columns). Linear regression lines have been overplotted. Shaded area shows the advocated abundance and uncertainty, $\log \epsilon_{\mathrm{C}}=8.47 \pm 0.02$.

ties in measuring these extremely weak lines to high precision and high accuracy.

The two rotational groups, $\mathrm{NH}|\Delta v|=0$ and $\mathrm{OH}|\Delta v|=0$, show rather strong trends in Fig. 4 and Fig. 5. These lines are in the far infrared, between 11300 and $15000 \mathrm{~nm}$ for $\mathrm{NH}$ and between 9400 and $12300 \mathrm{~nm}$ for $\mathrm{OH}$; i.e; further to the IR than the rovibrational lines of $\mathrm{CH}, \mathrm{NH}$ and $\mathrm{OH}$ (Table 1). Conse- quently, these infrared rotational lines form higher up in the atmosphere than the rovibrational lines (at Rosseland mean optical depths that are nearly 1 dex smaller), on account of the strong wavelength dependence of the dominant $\mathrm{H}^{-}$free-free continuous opacity. Thus the depicted trends may in part reflect inadequacies in the uppermost layers of the 3D model due to for example the lack of magnetic fields, and absence of a chromosphere. 

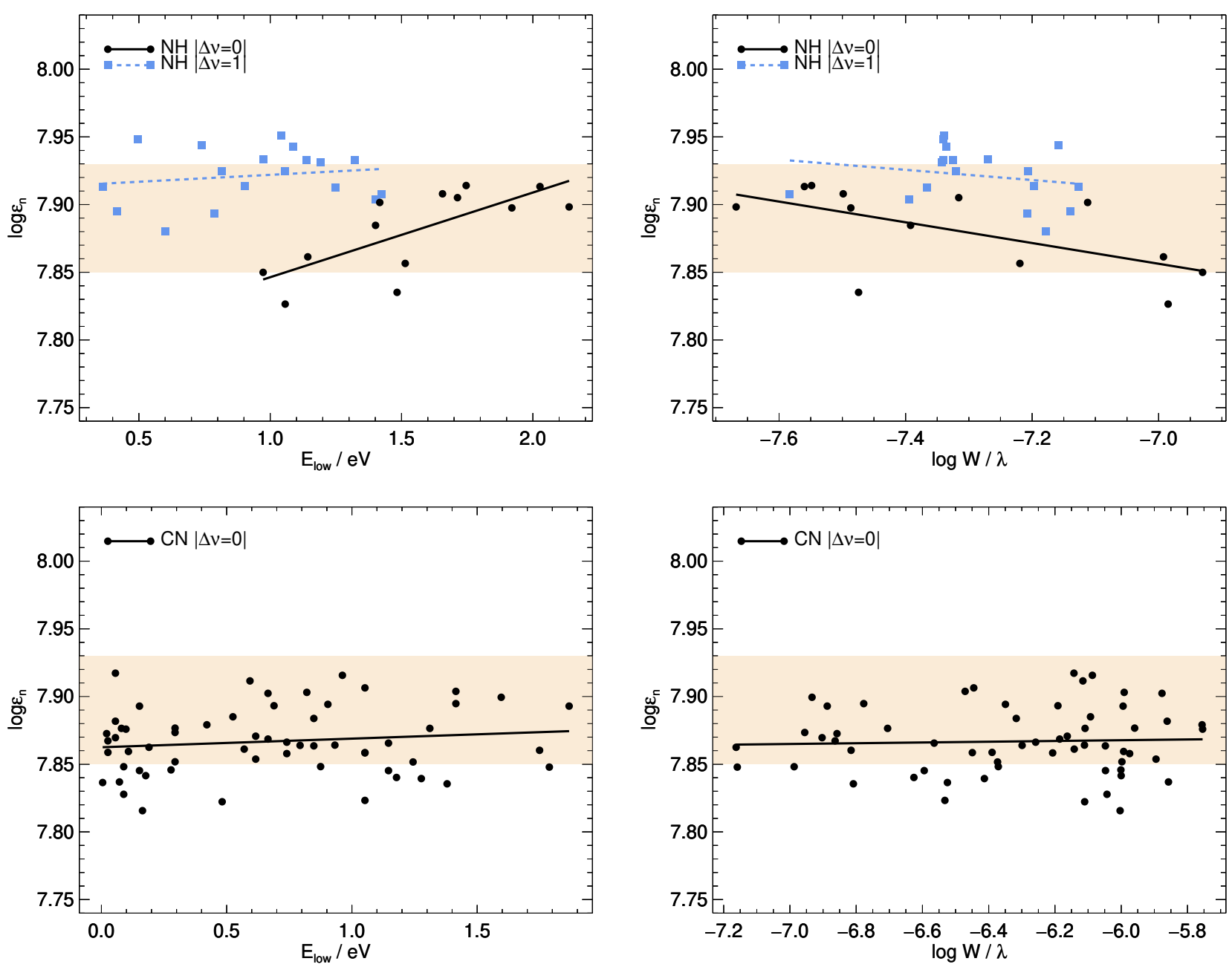

Fig. 4. Nitrogen abundances inferred from lines of $\mathrm{NH}$ and $\mathrm{CN}$ (rows), against excitation potential and reduced equivalent width (columns). Linear regression lines have been overplotted. Shaded area shows the advocated abundance and uncertainty, $\log \epsilon_{\mathrm{N}}=7.89 \pm 0.04$.

The $\mathrm{OH}|\Delta v|=0$ lines are stronger than the $\mathrm{NH}|\Delta v|=0$ lines (median equivalent widths of $5.6 \mathrm{pm}$ and $1.7 \mathrm{pm}$, respectively), and typically form at greater heights; accordingly, they display a more prominent trend relative to the scatter. In both cases, the results from the deeper-forming fainter lines tend to agree better with the rovibrational lines, as expected.

The figures provide some insight on the most and least reliable abundance indicators, for each molecule, and for each element. For carbon, the $\mathrm{C}_{2}$ Swan bands emerge as the most reliable indicators. They are absent of significant trends with line parameters (Fig. 3), and also have low sensitivity to the model atmosphere (Fig. 6). The ${ }^{12} \mathrm{C}^{16} \mathrm{O}$ lines are the least reliable ones, despite their low scatter. This is expected because of the temperature sensitivity of their formation, and thus their sensitivity to the model atmosphere as is reflected in Fig. 6. For nitrogen, NH and $\mathrm{CN}$ are found to have similar weight. Within $\mathrm{NH}$, the $|\Delta v|=1$ lines have the most weight, as the $|\Delta v|=0$ lines show more pronounced trends as discussed above. Finally for oxygen all three groups have similar sensitivities to the model atmosphere; the $\mathrm{OH}|\Delta v|=1$ lines are the most reliable, because of the noticeable trends in the $\mathrm{OH}|\Delta v|=0$ and $\mathrm{OH}|\Delta v|=2$ groups.

\subsection{Comparison with the $1 D$ models}

Typically, the results from the various 1D models are larger than those from the 3D model - in other words, the 3D effects are usually negative. For carbon, nitrogen, and oxygen respectively, the $\langle 3 \mathrm{D}\rangle$ results are $0.02,0.10$, and 0.10 dex larger; the ATMO and MARCS results are $-0.02,0.06$, and 0.06 dex larger, and the HM74 results are $0.08,0.15$, and 0.15 dex larger. This systematic effect is because of the enhanced formation of molecules in the cool pockets of gas that arise due to solar convection. It is particularly effective for the temperature-sensitive ${ }^{12} \mathrm{C}^{16} \mathrm{O}$ lines, as well as the $\mathrm{NH}|\Delta v|=0$ lines and to a lesser extent the $\mathrm{OH}|\Delta v|=0$ lines (Fig. 6) that form in the uppermost regions of the photosphere.

It is perhaps counter-intuitive that the results from the semiempirical HM74 model deviates quite strongly from the 3D model. This is related to the smaller temperature gradient of that model, with temperatures around $50 \mathrm{~K}$ larger in the lineforming region relative to the $\langle 3 \mathrm{D}\rangle$ model (Fig. 2), as previously discussed by Asplund et al. (2009). While the origin of this temperature difference is not completely clear, we can speculate on a few possible reasons for it. The HM74 model presented in Holweger \& Müller (1974) is an updated version of Holweger (1967), with the photospheric temperature stratification in the line-forming region determined primarily by fitting 

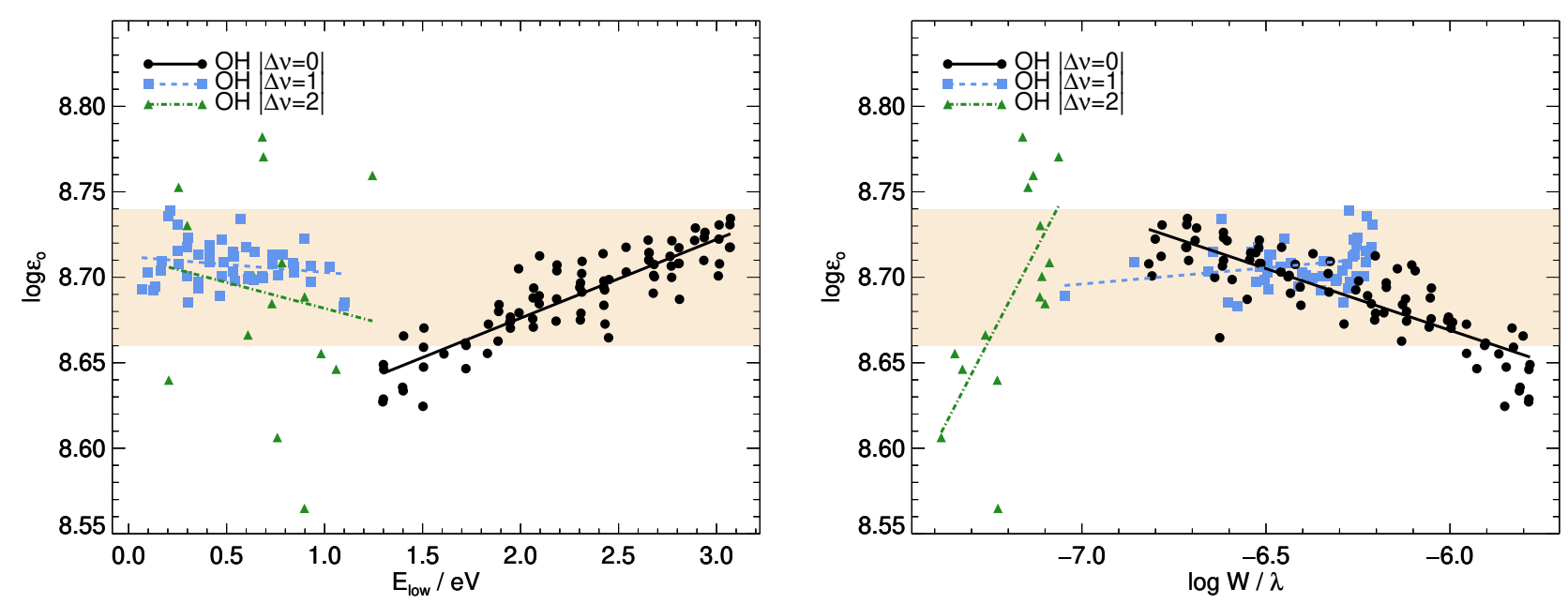

Fig. 5. Oxygen abundances inferred from lines of $\mathrm{OH}$, against excitation potential and reduced equivalent width (columns). Linear regression lines have been overplotted. Shaded area shows the advocated abundance and uncertainty, $\log \epsilon_{\mathrm{O}}=8.70 \pm 0.04$.

the centre-to-limb variations of the central intensities of some 900 atomic and ionic lines, under the assumption of LTE. Our measurements suggest that the line depths given by Holweger (1967) are slightly but systematically smaller than those in in the more recent and higher-quality Liège and Hamburg solar atlases employed here. This could be associated with difficulties in correcting for stray light and the lower spectral resolution of the spectrograph used by Holweger (1967), although systematic differences in the continuum normalisation could also play a role. Adopting shallower line depths in the construction of the HM74 model could at least partially explain the shallower temperature gradient and thus the larger estimate of the temperature in the line forming regions. The assumption of LTE for neutral species of low to moderate ionisation potential including $\mathrm{Mg}_{\mathrm{I}}$ and $\mathrm{Fe} \mathrm{I}$ may lead to a similar effect (non-LTE masking; Rutten \& Kostik 1982); although, this is compensated by their choice of high elemental abundances $\log \epsilon_{\mathrm{Mg}}=\log \epsilon_{\mathrm{Fe}}=7.60$ when determining the electron number densities. It can be noted that newer semi-empirical solar models of the quiet Sun such as those by Grevesse \& Sauval (1999) and Allende Prieto et al. (2001), have temperature structures more closely resembling that of the $\langle 3 \mathrm{D}\rangle$ model.

\subsection{Comparison with earlier studies of molecules}

The abundance values advocated in Sect. 3.3 generally agree well with those of Asplund et al. (2009), which was an updated analysis of Asplund et al. (2005b) for carbon and Asplund et al. (2004) for oxygen using an improved 3D solar model. There are, nevertheless, some puzzling discrepancies: the most prominent ones are for $\mathrm{CH}$ and $\mathrm{NH}$, which are around 0.03 to 0.05 dex larger here. This offset does not seem to be due mainly to the model atmosphere (being also apparent for the results from the 1D MARCS models), transition probabilities, nor the adopted equivalent widths. We speculate that incremental updates to the line formation code, and equation of state solver (including input data such as partition functions and equilibrium constants) could be the cause of the offset. While the average result for $\mathrm{OH}$ is consistent, it should be noted that the line-by-line results here do not show the strong non-linear trends apparent in Fig. 10 of Asplund et al. (2004).
The ${ }^{12} \mathrm{C}^{16} \mathrm{O}$ lines were not used to inform the carbon or oxygen abundances in Asplund et al. (2009). This is primarily because of their temperature sensitivity, although they may also be sensitive to departures from instantaneous chemical equilibrium (Uitenbroek 2000; Asensio Ramos et al. 2003); such effects are neglected in the present study. Nevertheless, elemental abundances via ${ }^{12} \mathrm{C}^{16} \mathrm{O}$ lines were also derived by Scott et al. (2006) (with the same 3D solar model as in Asplund et al. 2005b). Fixing $\log \epsilon_{\mathrm{O}}=8.66$ they obtained $\log \epsilon_{\mathrm{C}}=8.48$ and 8.40 from ${ }^{12} \mathrm{C}^{16} \mathrm{O}|\Delta v|=1$ lines of low and high excitation potential, respectively; and $\log \epsilon_{\mathrm{C}}=8.37$ from ${ }^{12} \mathrm{C}^{16} \mathrm{O}|\Delta v|=2$ lines. The line selection adopted here derives from theirs, albeit with updated transition probabilities from Li et al. (2015) that are much improved as discussed in Lyons et al. (2018). We find consistent results from all of these ${ }^{12} \mathrm{C}^{16} \mathrm{O}$ lines of around $\log \epsilon_{\mathrm{C}}=8.48 \mathrm{dex}$, as illustrated in Fig. 3. Test calculations suggest that the updates to the $3 \mathrm{D}$ model help to ensure congruous results from the different ${ }^{12} \mathrm{C}^{16} \mathrm{O}$ lines.

\subsection{Comparison with atomic results}

It is illuminating to compare the molecular results derived here with atomic results present in the literature. Recently, 3D nonLTE studies of C I (Amarsi et al. 2019; Li et al. 2021), N I (Amarsi et al. 2020a), and O I (Amarsi et al. 2018) lines have been carried out. These studies are based on the same model atmosphere as that used here. In addition, 3D LTE studies with 1D non-LTE corrections have been carried out for these species by Caffau et al. (2008, 2009, 2010, 2013, 2015), as well as full 3D non-LTE calculations of the O $777 \mathrm{~nm}$ triplet by Steffen et al. (2015) on an independent 3D solar model computed with the CO ${ }^{5}$ BOLD code (Freytag et al. 2012).

A comparison can be found in Asplund et al. (2021); the main findings are summarised here. Due to several different updates, in particular a systematic shift in the transition probabilities for $\mathrm{C}_{\mathrm{I}}$ (Li et al. 2021), the various carbon abundance indicators all now point to a value of around $8.46 \mathrm{dex}$, which is $0.03 \mathrm{dex}$ larger than the value advocated by Asplund et al. (2009). For oxygen, the agreement is also very satisfactory, with the [O I] lines and $\mathrm{O}_{\mathrm{I}}$ lines indicating on average slightly higher and lower 


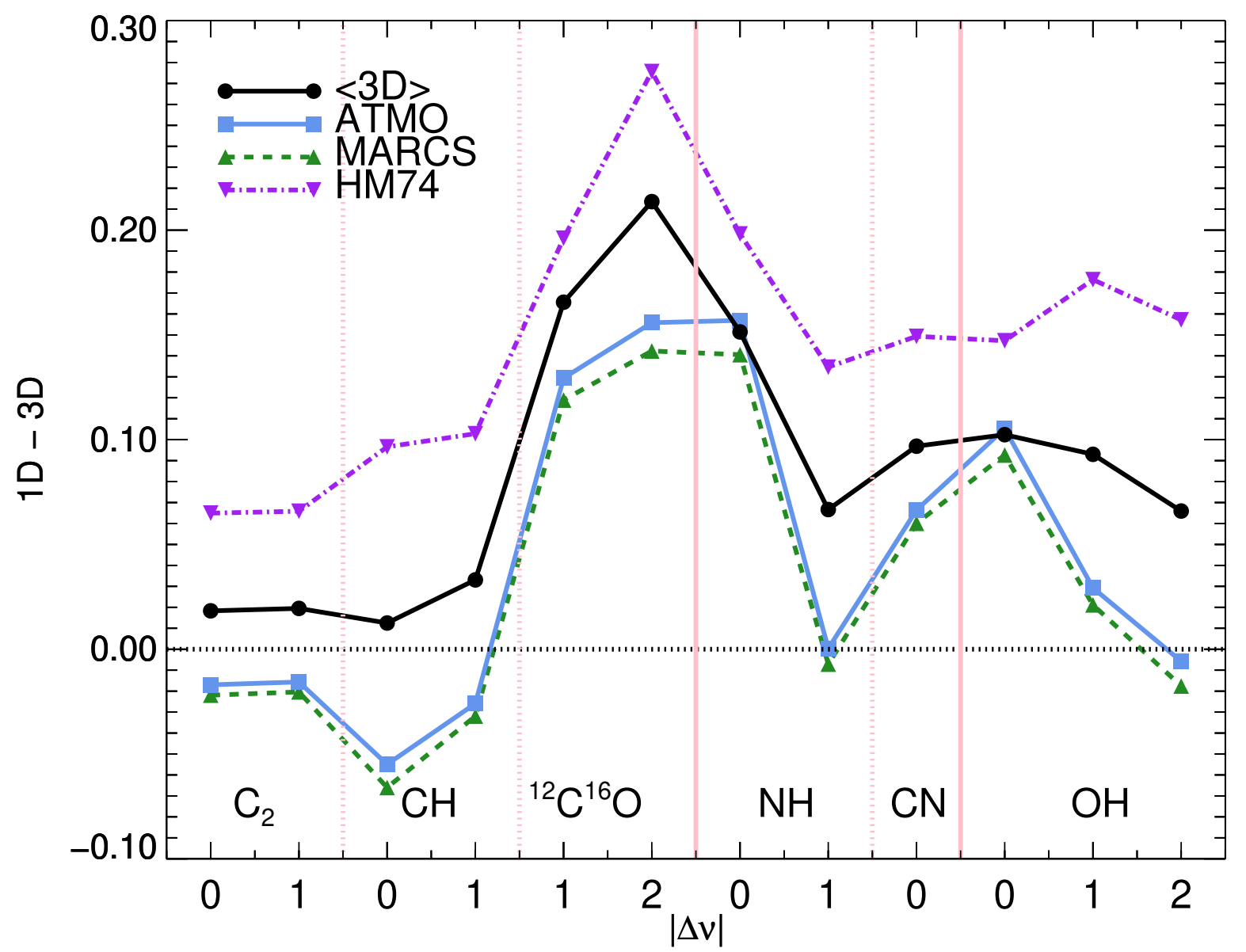

Fig. 6. Difference between 1D and 3D elemental abundances for the different groups in Table 1.

results by around 0.02 dex, compared to the molecular result of 8.70 dex found here.

For nitrogen, however, there is a prominent discrepancy with the atomic lines. With the present 3D model, the $\mathrm{N}_{\mathrm{I}}$ lines give $\log \epsilon_{\mathrm{N}}=7.77 \pm 0.05$. This is significantly lower than the present molecular result of $7.89 \pm 0.04 \mathrm{dex}$; a difference of $0.12 \mathrm{dex}$. The origin of this problem is unclear. Systematic errors in the transition probabilities or other molecular data cannot be ruled out, but seem unlikely as discussed in Asplund et al. (2021). The atomic and molecular lines have opposite temperature sensitivities; consequently, errors in the temperature structure of the 3D model could help explain the discrepancy, although the large changes needed would worsen the agreement between the various carbon and oxygen abundance indicators, as well as the excellent agreement with various other solar observables as studied by Pereira et al. (2013) for the previous generation of the 3D STAGGER model. If this is indeed the case, the true solar nitrogen abundance would be close to the mean result from the atoms and molecules: $\log \epsilon_{\mathrm{N}}=7.83$, as advocated in Asplund et al. (2021). It can be noted that the various $1 \mathrm{D}$ models show even larger discrepancies: for instance, the HM74 model indicates a difference of $0.18 \mathrm{dex}$, with $\log \epsilon_{\mathrm{N}}=7.86$ from the $\mathrm{N}_{\mathrm{I}}$ lines (unpublished results from Amarsi et al. 2020a), and $\log \epsilon_{\mathrm{N}}=8.04$ from the molecules (Table 3).

More recently, Bergemann et al. (2021) have studied of the O $777 \mathrm{~nm}$ triplet lines and the [O I] $630 \mathrm{~nm}$ line, using 13 snap- shots of the 3D model solar atmosphere from the STAGGER-grid (Collet et al. 2011; Magic et al. 2013). This 3D model is similar to that employed in Amarsi et al. (2018, 2019, 2020a) as well as in the present study: it was computed with the STAGGER code, employing the standard solar chemical composition of Asplund et al. (2009); and the 13 snapshots have a mean effective temperature of $5773 \mathrm{~K}$. The authors employed column-bycolumn non-LTE radiative transfer (the so-called 1.5D non-LTE approach); Fig. 6 of that paper suggests that, at least in this particular case, this approximation corresponds to errors that are much less than 0.01 dex, compared to the more realistic 3D nonLTE approach. From the $\mathrm{O}_{\mathrm{I}}$ and $\left[\mathrm{O}_{\mathrm{I}}\right]$ features they obtained $\log \epsilon_{\mathrm{O}}=8.74 \pm 0.03$ and $\log \epsilon_{\mathrm{O}}=8.77 \pm 0.05$, respectively, finally advocating $\log \epsilon_{\mathrm{O}}=8.75 \pm 0.03$. This is slightly larger than one standard deviation away from our own measurements from the same features: $8.69 \pm 0.03$ dex and $8.70 \pm 0.05$ dex respectively (Amarsi et al. 2018; Asplund et al. 2021). Given the importance of the solar oxygen abundance on the solar modelling problem, we briefly discuss below from where these differences may originate.

For the O I $777 \mathrm{~nm}$ triplet lines, $0.026 \mathrm{dex}$ of the difference can be explained by the choice of transition probabilities. Bergemann et al. (2021) adopted the recent values of Civiš et al. (2018) that are based on quantum defect theory (e.g. Seaton 1966), averaged together with those of Hibbert et al. (1991) that are based on the (more sophisticated) configuration interaction (CI) approach 
(as implemented in the CIV3 code; Hibbert 1975). In contrast, Amarsi et al. (2018) used only the latter data set. For the [O I] $630 \mathrm{~nm}$ line, most of the discrepancy probably reflects the inconsistent treatment of the $\mathrm{N}_{\mathrm{I}}$ blend. Bergemann et al. (2021) assumed $\log \epsilon_{\mathrm{Ni}}=6.23$ (Lodders 2003), and subsequently corrected the strength of the blend for absolute 1.5D non-LTE effects, rather than deriving a nickel abundance consistent with their model atmosphere and radiative transfer approach using other, clean Ni I lines (see the discussion in Scott et al. 2009). Effectively, Bergemann et al. (2021) adopted a 0.05 dex smaller nickel contribution to the $630 \mathrm{~nm}$ feature compared to Asplund et al. (2021), which translates to a 0.03 dex larger inferred oxygen abundance. The remaining discrepancies may be due to differences between their new FTS data, and the Liège and Hamburg solar atlases employed in Asplund et al. (2021). They may also be partly due to systematic differences in the 3D non-LTE radiative transfer codes employed.

\subsection{Advocated solar elemental abundances and metallicity}

It is preferable to fold in both atomic and molecular results to obtain final estimates on the elemental abundances, in particular for nitrogen. These advocated values can be found in Asplund et al. (2021): $\log \epsilon_{\mathrm{C}}=8.46, \log \epsilon_{\mathrm{N}}=7.83, \log \epsilon_{\mathrm{O}}=8.69$. Nevertheless, it is illuminating to consider the impact on the solar metallicity when adopting the carbon, nitrogen, and oxygen abundances inferred here, these values being slightly larger than the atom-molecule averages. With the chemical mixture of Asplund et al. (2021), the metal mass fraction of the solar surface is $Z=0.0139 \pm 0.0006$; or, relative to the hydrogen mass fraction, $Z / X=0.0187 \pm 0.0009$. With the molecular abundances determined here, these values increase to $Z=0.0142$ and $Z / X=0.0191$, where $45 \%$ of the difference is due to the 0.01 dex increase in oxygen abundance, $35 \%$ is due to the relatively large 0.06 dex increase in nitrogen abundance, and just $20 \%$ is due to the 0.01 dex increase in carbon abundance. The differences thus remain well within the stipulated uncertainties.

\section{Conclusion}

We have analysed 408 molecular lines of carbon, nitrogen, and oxygen in the solar intensity spectrum using LTE line formation calculations in a $3 \mathrm{D}$ radiative-hydrodynamic simulation of the photosphere. From this analysis, advocated elemental abundances of $\log \epsilon_{\mathrm{C}}=8.47 \pm 0.02, \log \epsilon_{\mathrm{N}}=7.89 \pm 0.04$, and $\log \epsilon_{\mathrm{O}}=8.70 \pm 0.04$ were obtained. For carbon and oxygen, these are in good agreement with earlier 3D non-LTE analyses of atomic diagnostics (Amarsi et al. 2018, 2019; Li et al. 2021; Asplund et al. 2021). However, for nitrogen there is a discrepancy approaching $3 \sigma$ of $0.12 \mathrm{dex}$, compared to the results presented in Amarsi et al. (2020a).

The elemental abundances of these elements remain relevant in the solar modelling problem. Although oxygen dominates the opacity in the most discrepant region, carbon and nitrogen are also relevant because they are all mainly present as neutral atoms in the solar photosphere, their molecules are closely coupled together via ${ }^{12} \mathrm{C}^{16} \mathrm{O}$ and $\mathrm{CN}$, and they are similarly depleted in meteorites (Lodders et al. 2009; Palme et al. 2014) and must therefore be inferred via spectroscopic methods. The solar oxygen abundance determined here, consistent with the atomic results, is just 0.01 dex larger than that advocated in Asplund et al. (2009) and unfortunately does little to alleviate the solar modelling problem. The carbon and nitrogen abundances determined here are 0.03 and 0.06 dex larger than the values advocated by
Asplund et al. (2009). Although this may only slightly reduce the discrepancy with helioseismology, this revision does indicate that it may be worthwhile to continue improving the atomic and molecular data as well as the model atmospheres and line formation methods.

Acknowledgements. We are grateful to Matthias Steffen for providing a detailed and insightful referee report, which led to a number of improvements to this manuscript. We thank T. Masseron and C. Sneden for their assistance with compiling the molecular data. AMA acknowledges support from the Swedish Research Council (VR 2016-03765 and 2020-03940). MA acknowledges funding from the Australian Research Council through a Laureate Fellowship (FL110100012) and a Discovery Project (DP150100250). This research was supported by computational resources provided by the Australian Government through the National Computational Infrastructure (NCI) under the National Computational Merit Allocation Scheme and the ANU Merit Allocation Scheme (project y89).

\section{References}

Abrams, M. C., Goldman, A., Gunson, M. R., Rinsland, C. P., \& Zander, R. 1996, Appl. Opt., 35, 2747

Allende Prieto, C., Barklem, P. S., Asplund, M., \& Ruiz Cobo, B. 2001, ApJ, 558,830

Amarsi, A. M., Barklem, P. S., Asplund, M., Collet, R., \& Zatsarinny, O. 2018, A\&A, 616, A89

Amarsi, A. M., Barklem, P. S., Collet, R., Grevesse, N., \& Asplund, M. 2019, A\&A, 624, A111

Amarsi, A. M., Grevesse, N., Grumer, J., et al. 2020a, A\&A, 636, A120

Amarsi, A. M., Lind, K., Osorio, Y., et al. 2020b, A\&A, 642, A62

Asensio Ramos, A., Trujillo Bueno, J., Carlsson, M., \& Cernicharo, J. 2003, ApJ, 588, L61

Asplund, M. 2005, ARA\&A, 43, 481

Asplund, M., Amarsi, A. M., \& Grevesse, N. 2021, arXiv e-prints, arXiv:2105.01661

Asplund, M., Grevesse, N., \& Sauval, A. J. 2005a, in Astronomical Society of the Pacific Conference Series, Vol. 336, Cosmic Abundances as Records of Stellar Evolution and Nucleosynthesis, ed. T. G. Barnes, III \& F. N. Bash, 25 Asplund, M., Grevesse, N., Sauval, A. J., Allende Prieto, C., \& Blomme, R. 2005b, A\&A, 431, 693

Asplund, M., Grevesse, N., Sauval, A. J., Allende Prieto, C., \& Kiselman, D. 2004, A\&A, 417, 751

Asplund, M., Grevesse, N., Sauval, A. J., \& Scott, P. 2009, ARA\&A, 47, 481

Asplund, M., Nordlund, A., Trampedach, R., Allende Prieto, C., \& Stein, R. F. 2000, A\&A, 359, 729

Ayres, T. R., Lyons, J. R., Ludwig, H.-G., Caffau, E., \& Wedemeyer-Böhm, S. 2013, ApJ, 765, 46

Ayres, T. R. \& Wiedemann, G. R. 1989, ApJ, 338, 1033

Bahcall, J. N., Serenelli, A. M., \& Basu, S. 2005, ApJ, 621, L85

Bailey, J. E., Nagayama, T., Loisel, G. P., et al. 2015, Nature, 517, 56

Barklem, P. S. \& Collet, R. 2016, A\&A, 588, A96

Basu, S. \& Antia, H. M. 2008, Phys. Rep., 457, 217

Bergemann, M., Hoppe, R., Semenova, E., et al. 2021, MNRAS

Brooke, J. S. A., Bernath, P. F., Schmidt, T. W., \& Bacskay, G. B. 2013, J. Quant. Spectr. Rad. Transf., 124, 11

Brooke, J. S. A., Bernath, P. F., \& Western, C. M. 2015, J. Chem. Phys., 143, 026101

Brooke, J. S. A., Bernath, P. F., Western, C. M., et al. 2016, J. Quant. Spectr. Rad. Transf., 168, 142

Brooke, J. S. A., Bernath, P. F., Western, C. M., van Hemert, M. C., \& Groenenboom, G. C. 2014a, J. Chem. Phys., 141, 054310

Brooke, J. S. A., Ram, R. S., Western, C. M., et al. 2014b, ApJS, 210, 23

Buldgen, G., Salmon, S., \& Noels, A. 2019, Frontiers in Astronomy and Space Sciences, 6, 42

Caffau, E., Ludwig, H.-G., Bonifacio, P., et al. 2010, A\&A, 514, A92

Caffau, E., Ludwig, H.-G., Malherbe, J.-M., et al. 2013, A\&A, 554, A126

Caffau, E., Ludwig, H.-G., Steffen, M., et al. 2008, A\&A, 488, 1031

Caffau, E., Ludwig, H.-G., Steffen, M., et al. 2015, A\&A, 579, A88

Caffau, E., Maiorca, E., Bonifacio, P., et al. 2009, A\&A, 498, 877

Christensen-Dalsgaard, J. 2021, Living Reviews in Solar Physics, 18, 2

Christensen-Dalsgaard, J., di Mauro, M. P., Houdek, G., \& Pijpers, F. 2009, A\&A, 494, 205

Civiš, S., Kubelík, P., Ferus, M., et al. 2018, ApJS, 239, 11

Collet, R., Magic, Z., \& Asplund, M. 2011, Journal of Physics Conference Series, 328,012003

Collet, R., Nordlund, Ã., Asplund, M., Hayek, W., \& Trampedach, R. 2018, MNRAS, 475, 3369 
Delbouille, L. \& Roland, C. 1995, in Astronomical Society of the Pacific Conference Series, Vol. 81, Laboratory and Astronomical High Resolution Spectra, ed. A. J. Sauval, R. Blomme, \& N. Grevesse, 32

Delbouille, L., Roland, G., Brault, J., \& Testerman, L. 1981, Photometric Atlas of the Solar Spectrum from 1,850 to $10,000 \mathrm{~cm}-1$ (Kitt Peak National Observatory)

Delbouille, L., Roland, G., \& Neven, L. 1973, Atlas photometrique du spectre solaire de [lambda] 3000 a [lambda] 10000 (Liege: Universite de Liege, Institut d'Astrophysique)

Doerr, H.-P., Vitas, N., \& Fabbian, D. 2016, A\&A, 590, A118

Freytag, B., Steffen, M., Ludwig, H.-G., et al. 2012, Journal of Computational Physics, 231, 919

Grevesse, N., Lambert, D. L., Sauval, A. J., et al. 1990, A\&A, 232, 225

Grevesse, N., Lambert, D. L., Sauval, A. J., et al. 1991, A\&A, 242, 488

Grevesse, N. \& Sauval, A. J. 1999, A\&A, 347, 348

Grevesse, N., Scott, P., Asplund, M., \& Sauval, A. J. 2015, A\&A, 573, A27

Gustafsson, B., Edvardsson, B., Eriksson, K., et al. 2008, A\&A, 486, 951

Hase, F., Wallace, L., McLeod, S. D., Harrison, J. J., \& Bernath, P. F. 2010, J. Quant. Spectr. Rad. Transf., 111, 521

Hayek, W., Asplund, M., Collet, R., \& Nordlund, A. 2011, A\&A, 529, A158

Hibbert, A. 1975, Computer Physics Communications, 9, 141

Hibbert, A., Biemont, E., Godefroid, M., \& Vaeck, N. 1991, J. Phys. B, 24, 3943

Hinkle, K. H. \& Lambert, D. L. 1975, MNRAS, 170, 447

Holweger, H. 1967, ZAp, 65, 365

Holweger, H., Gehlsen, M., \& Ruland, F. 1978, A\&A, 70, 537

Holweger, H. \& Müller, E. A. 1974, Sol. Phys., 39, 19

Kiselman, D. 1993, A\&A, 275, 269

Lambert, D. L. 1978, MNRAS, 182, 249

Li, G., Gordon, I. E., Rothman, L. S., et al. 2015, ApJS, 216, 15

Li, W., Amarsi, A. M., Papoulia, A., Ekman, J., \& Jönsson, P. 2021, MNRAS, 502,3780

Lodders, K. 2003, ApJ, 591, 1220

Lodders, K., Palme, H., \& Gail, H.-P. 2009, Landolt Börnstein, 44

Lyons, J. R., Gharib-Nezhad, E., \& Ayres, T. R. 2018, Nature Communications, 9, 908

Magic, Z., Collet, R., Asplund, M., et al. 2013, A\&A, 557, A26

Masseron, T., Plez, B., Van Eck, S., et al. 2014, A\&A, 571, A47

Meléndez, J. 2004, ApJ, 615, 1042

Mondet, G., Blancard, C., Cossé, P., \& Faussurier, G. 2015, ApJS, 220, 2

Neckel, H. 1999, Sol. Phys., 184, 421

Neckel, H. \& Labs, D. 1984, Sol. Phys., 90, 205

Nordlund, A., Stein, R. F., \& Asplund, M. 2009, Living Reviews in Solar Physics, 6,2

Palme, H., Lodders, K., \& Jones, A. 2014, Solar System Abundances of the Elements, ed. A. M. Davis, Vol. 2 (Elsevier), 15-36

Pereira, T. M. D., Asplund, M., Collet, R., et al. 2013, A\&A, 554, A118

Prša, A., Harmanec, P., Torres, G., et al. 2016, AJ, 152, 41

Rutten, R. J. \& Kostik, R. I. 1982, A\&A, 115, 104

Scott, P., Asplund, M., Grevesse, N., Bergemann, M., \& Sauval, A. J. 2015a, A\&A, 573, A26

Scott, P., Asplund, M., Grevesse, N., \& Sauval, A. J. 2009, ApJ, 691, L119

Scott, P., Grevesse, N., Asplund, M., et al. 2015b, A\&A, 573, A25

Scott, P. C., Asplund, M., Grevesse, N., \& Sauval, A. J. 2006, A\&A, 456, 675

Seaton, M. J. 1966, Proceedings of the Physical Society, 88, 801

Serenelli, A. M., Basu, S., Ferguson, J. W., \& Asplund, M. 2009, ApJ, 705, L123

Steffen, M., Prakapavičius, D., Caffau, E., et al. 2015, A\&A, 583, A57

Tennyson, J. \& Yurchenko, S. N. 2012, MNRAS, 425, 21

Tennyson, J., Yurchenko, S. N., Al-Refaie, A. F., et al. 2016, Journal of Molecular Spectroscopy, 327, 73

Uitenbroek, H. 2000, ApJ, 536, 481

Wallace, L. \& Livingston, W. 2003, An atlas of the solar spectrum in the infrared from 1850 to $9000 \mathrm{~cm}-1$ (1.1 to 5.4 micrometer) (Tucson: National Solar Observatory, National Optical Astronomy Observatory, NSO Technical Report) 